\title{
Acerca del origen de la huerta de Orihuela y la explotación de las zonas húmedas del Bajo Segura entre los siglos VII y XI. Respuesta a M. Barceló
}

\author{
Sonia Gutiérrez Lloret
}

Estas reflexiones quieren ser una respuesta a las cuestiones planteadas por M. Barceló acerca de dos artículos escritos por mí -publicado el primero en Arbor (1995 a) y el segundo en este mismo número de la revista Arqueología y Territorio Medieval-, en un trabajo cuyo texto me ha sido facilitado por la dirección de dicha revista a fin de ejercer el derecho a contrarréplica.' También quiero agradecer a la misma la posibilidad que me brinda y que favorece el intercambio de opiniones fundamental en toda disciplina científica. Esta dimensión dialogante del proceso de reflexión histórica convierte la crítica en un ejercicio científico de humildad y reflexión sobre lo que nos dicen los otros, apartándola, como recientemente reclamaba Javier Arce en otro foro de debate de similares caraterísticas, de los cenáculos y de la arbitrariedad (ARCE, 1993, p. 24).

Sin embargo, no puedo dejar de manifestar mi perplejidad ante unas maneras desabridas que no considero propias del discurso científico y que me disgustan doblemente por proceder de un historiador de la talla intelectual de quien suscribe. En primer lugar, la crítica de $M$. Barceló me parece desmesurada hasta en su mismo título, porque raramente un comentario "acerca de nada" genera tantas y tan densas páginas, aunque, como señaló acertadamente Ricardo Olmos, en todas las críticas pueden surgir excelentes ideas, dignas de atención y potenciadoras de fructíferas reflexiones
(OLMOS, 1993, p. 23). De otro lado, la crítica puede ser contundente, irónica e incluso destructiva aunque éstas raramente sean fértiles-, pero si quiere ser científica debe ser siempre sincera y honesta, y cuando reclamo honestidad en una crítica a mi trabajo, lo que reclamo es que se rebata lo que yo he dicho directamente y no aquello que el crítico supone que pienso pero que no digo -difícil y estéril "deconstrucción" aquella que se ejerce sobre pensamientos que se suponen o imaginan- o, peor aún, sobre lo que supuestamente hago decir a colegas con los que en muchos casos mantengo además un excelente diálogo -que no siempre quiere decir acuerdo- por demás fluido y directo. Conviene, pues, recordar que los investigadores no tenemos derecho a la mala fe, como no hace mucho señalaba el propio M. Barceló (1993, p. 49). Por este motivo aspiro a que se analicen mis hipótesis de acuerdo con su desarrollo científico y me parece cuando menos incorrecto que se juzguen por la pertinencia o no de mis lecturas, puesto que el citar aquello que uno considera necesario, útil, interesante o sugerente es un acto de libertad de pensamiento sobre el que no puede ni debe existir ningún principio de autoridad.

Aclarados convenientemente estos aspectos de forma, que considero cruciales, quisiera comentar con brevedad ciertas cuestiones conceptuales discutidas por M. Barceló. En su trabajo el autor plantea una réplica ante lo que

I BARCELÓ, M.: "Acerca de nada. Consideraciones sobre dos artículos de S. Gutiérrez", presentado al coloquio Historia y Medio Físico (Agricultura y regadío en Al-Andalus, Síntesis y problemas), Almería, Junio 1995, y publicado en este mismo número de la revista Arqueología y Territorio Medieval, pp. 21-36 
considera una "vulneración grave y arbitraria" 2 de un sistema conceptual por él elaborado o de ciertas nociones del mismo, que al parecer utilizo de forma perversa, puesto que oculto mi conocimiento de los trabajos donde se exponen, e interesada ya que al no mencionar su procedencia intelectual supuestamente me las atribuyo; tal es el caso de las nociones de "diseño" o "línea de rigidez". Realmente lamento haber enojado tanto a M. Barceló con algo que en principio debería ser motivo de orgullo, puesto que representa el reconocimiento pleno de un científico, su aspiración máxima, e indica que sus conceptuaciones han sido globalmente asumidas por la comunidad científica, sin ser ya necesario citar a Newton cada vez que se hace referencia a la gravedad -si se me permite un paralelo jocoso-, o a Pierre Toubert y a Guillem Rossellò, cada vez que se menciona o utiliza el concepto de "incastellamento", formulado por el primero, o los de ataifor, redoma o cualquiera de los otros términos acuñados por el segundo, de uso corriente en la terminología histórica y arqueológica.

No obstante, si el autor así lo prefiere tomo cumplida nota y lo tendré en cuenta para futuros trabajos. Lo que no acepto es que deduzca de ese hecho mi desconocimiento de los mismos, mi subrepticia intención de atribuirme sus descubrimientos -nada más lejos de mi intención ya que es evidente, y así nos consta, que son suyos- ni menos aún de silenciarlos interesadamente. No insistiré sobre este aspecto porque creo que cualquier lector a quien interese el debate que nos ocupa, podrá comprobar, recurriendo a mi bibliografía, que no sólo cito abundantemente a $M$. Barceló, sino que su obra constituye, como es notorio, una fuente de reflexión básica y una referencia obligada de mis propios trabajos. Como no es cuestión de referir pormenorizadamente estas menciones remito únicamente a dos de los más recientes (GUTIÉRREZ LLORET, 1994 y 1995 b).

Con independencia de estas consideraciones, la crítica de M. Barceló incluye dos niveles distintos de reflexión: el que afecta a los princi- pios generales de la arqueología hidráulica y a sus aplicaciones y el que se refiere al análisis histórico y social que se deriva de la investigación arqueológica que vengo desarrollando en el sureste de al-Andalus. Comenzaré por el segundo sin ánimo de extenderme sino de dar únicamente una respuesta concisa. M. Barceló cuestiona una serie de hipótesis sobre la formación de la sociedad andalusí en Tudmìr durante la época emiral, que se inscriben en una línea de interpretación histórica desarrollada en otras regiones meridionales de alAndalus, especialmente en tierras de Andalucía oriental. Su análisis incide en la supuesta debilidad de la argumentación histórica y arqueológica que explica procesos sociales tan importantes como la "huida" de ciertos grupos urbanos y rústicos -que no creo haber calificado nunca de "masiva"-, la aparición y proliferación entre los siglos VI y IX de asentamientos en zonas elevadas y montuosas, protegidos por sus propias condiciones topográficas, el desarrollo de asentamientos en las inmediaciones de áreas pantanosas o los procesos de despoblamiento y transformación topográfica de las ciudades, arqueológicamente constatados; de otro lado, sugiere que tales hipótesis se sustentan únicamente en una argumentación circular de citas concatenadas. Pues bien, estos aspectos no se desarrollaron en los trabajos objeto de la crítica de $M$. Barceló porque no era ésta su finalidad, sino -y no conviene olvidarlo- el estudio arqueológico de la explotación de las zonas húmedas del Bajo Segura de un lado y los arcaduces de otro, refiriéndome por tanto únicamente a las publicaciones más recientes y sintéticas como la mencionada de M. Acién (1994).

Sin embargo, éste y otros argumentos han sido desarrollados en numerosos trabajos previos y posteriores a mi tesis doctoral (1988 a y b, 1992, 1993 a y b, 1994, 1995 b). M. Barceló los conoce perfectamente, puesto que se han abordado en el marco de coloquios y reuniones donde hemos tenido ocasión de discutirlos conjuntamente; de hecho, el esquema propuesto para explicar la formación de la sociedad islámica en Tudmir fue presentado de forma orgánica por

\footnotetext{
2 La cita de M. Barceló corresponde a su artículo "Acerca de nada...", página 28 del texto que me ha sido remitido por la revista.
} 
vez primera en un curso sobre la islamización de al-Andalus, celebrado en Málaga en 199|, y vuelto a discutir de nuevo en Córdoba en el marco de las Segundas Jornadas sobre Madinat al-Zahrā', donde fue publicado con posterioridad (1994) incluyendo algunas de las acertadas observaciones que tuvo la amabilidad de hacerme sobre el original el propio M. Barceló.

En esta misma línea me parece cuando menos parcial saldar las referencias a los mecanismos de integración o absorción de las poblaciones indígenas que supusieron los matrimonios mixtos o las implicaciones del asentamiento ŷundí, con una referencia a los trabajos clásicos de J. Ribera, R. Menéndez Pidal o C. Sánchez Albornoz. De esta forma, se obvian las investigaciones posteriores en torno al problema, suscitadas a partir de la obra fundamental de P. Guichard (1976) y desarrolladas con posterioridad por él mismo (1980 y 1985) así como por P. Chalmeta (1988), M. Acién (1984, 1989, 1994, e. p.) O E. Manzano (1993, e. p.), entre otros, cuyos planteamientos ideológicos sobre la islamización social están en las antípodas de los que alumbraron a los autores más señeros del "medievalismo tradicional español". Ciertamente P. Guichard expuso de forma clara e incontestable que "la sociedad árabe-musulmana tradicional" se basa en la endogamia preferencial -patrilineal y en menor medida matrilineal-, que favorece la "segmentarización social" en "células de parentesco, a la vez agnáticas y endogámicas" (GUICHARD, 1976, cap. I, esp. pp. 60-64), pero también señaló contundentemente que en los primeros siglos de al-Andalus existieron "dos sociedades yuxtapuestas y claramente diferenciadas: la indígena y la sociedad árabe-bereber", caracterizadas por distintas organizaciones sociales. Así, frente a los linajes indígenas que practicaban la exogamia, la tendencia a la endogamia de los conquistadores les aseguraba "la permanencia en el tiempo, la cohesión del linaje y una notable capacidad de expansión" (GUICHARD, 1976, p. 239).

Es evidente -en parte porque lo estudió P. Guichard (1976, 192-97; 1985, pp. 58-59)- que el matrimonio exogámico del ŷundí CAbd alŶabbār Ibn Nadīir con la hija de Teodomiro significó, entre otras cosas, la absorción del linaje de este personaje visigodo, hasta el punto de que sus descendientes -los conocidos y ricos Banū Jatțāb- conformaron rápidamente un linaje agnaticio sin demasiada conciencia de su origen materno. Pero lo que nos interesa ahora son precisamente las "otras" implicaciones de ese matrimonio respecto al tema que nos ocupa: el de la instalación de los ŷundíes y, evidentemente, de sus familias (GUICHARD, 1976, p. 197 y ss.). En primer lugar, Teodomiro -al que hemos de suponer miembro de la "aristocracia" visigoda fundiaria con capacidad efectiva de acordar una capitulacióncedió como dote dos propiedades rústicas situadas en el Bajo Segura. Este dato refuerza la hipótesis de un asentamiento rural de los linajes orientales (de procedencia egipcia en el territorio de Tudmir) en explotaciones agrícolas sobre las que la aristocracia mantenía un cierto control y que comenzamos a reconocer arqueológicamente, al igual que ocurre en otras regiones del sur de al-Andalus. De otro lado, la absorción del linaje de Teodomiro y seguramente de su patrimonio fundiario, como indicó P. Guichard (1976, p. 196), no supuso la inmediata pérdida de poder de otros sectores de la aristocracia visigoda, en clara connivencia con los ŷundíes. Esta sólida alianza se ejemplifica en el conocido episodio de la multa de 27.000 sueldos exigidos por Abū alJatțār a Atanagildo -sucesor, que no hijo, de Teodomiro en razón de su poder económicoy el pago de la misma efectuado por los ŷundíes en apenas tres días (GUTIÉRREZ LLORET, e. p.).

Asimismo es necesario aclarar que la forma en que cito a T. Schiøler (1973) es correcta y dice lo que dice, esto es, que los únicos paralelos -y hablamos de morfologíaque he encontrado para los arcaduces estudiados son algunas piezas egipcias publicadas por este autor, y si algún lector deduce de esto que Schiøler argumenta un posible origen egipcio de los arcaduces andalusíes, no tiene más que acudir a la obra citada para comprobar que está en un error, entre otras cosas porque los arcaduces alicantinos no habían sido exhumados cuando T. Schiøler escribió su libro. Tampoco he postulado la procedencia egipcia del sistema de anclaje de 
los arcaduces estudiados en el Bajo Segura, limitándome a señalar que egipcios son los paralelos conocidos, lo cual no pretende ser un argumento sino una mera constatación tipológica, sobre cuyos límites como argumentación histórica creo haber advertido yo misma. Resulta obvio, por tanto, que el recurso a un mero paralelismo formal -el de los arcaduces alicantinos con otros egipcios modernos- para probar el establecimiento de los ŷundíes egipcios en las alquerías del Bajo Segura es, a más de "abusivo" como indica M. Barceló, absolutamente innecesario, puesto que su origen egipcio lo conocemos por las fuentes árabes y la caracterización del poblamiento la proporciona la arqueología. En este sentido, debe recordarse que el origen indígena del poblamiento en esta región no depende, como parece entender M. Barceló, de una mención textual del siglo $\mathrm{XI}$ transmitida por

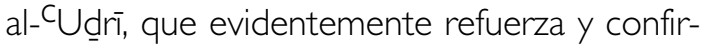
ma la argumentación arqueológica, sino del estudio sistemático del registro arqueológico de los siglos $\mathrm{VII}$ a $\mathrm{X}$ en dichos asentamientos, así como en otros muchos del sureste de alAndalus, cuyos materiales arqueológicos son de todos conocidos y están sobradamente publicados. $^{3}$

Por otra parte, el "proceso de uniformización tecnológica" al que me refiero en éste y otros trabajos previos se sitúa en la línea de constatación arqueológica que M. Barceló reclamaba precisamente en su trabajo de 1989: "Convendría, en este sentido, conducir a la investigación arqueológica a comprobar cómo esta pauta (de asentamiento-residencia y espacio hidráulico) y la tecnología que comporta se difunde en al-Andalus y es aceptada por grupos campesinos indígenas, no árabes y no bereberes" (BARCELÓ, 1989, XXXII); en el caso que nos ocupa es precisamente el estudio de las áreas de residencia y de los arcaduces que en ellas se documentan, lo que nos permite proponer la existencia de espacios hidráulicos, aunque no conozcamos su organización espacial o sus estructuras. Lo que sí podemos apreciar es que la síntesis de procesos tecnológicos, en expresión del propio M. Barceló y en respuesta a su demanda, que supone la generalización de la noria, había comenzado ya en el siglo VIII -por la datación arqueológica de los contextos donde aparecen por vez primera estas piezas- y que en consecuencia estaba ya muy avanzada en el siglo $X$, como M. Barceló apunta (1989, XXXIII) y como se aprecia además en otros elementos del registro arqueológico (BARCELÓ, 1992, p. 248; GUTIÉRREZ LLORET, 1993 b, p. 60).

Esta última consideración da paso al segundo nivel de reflexión planteado por $M$. Barceló, el que afecta a los principios generales de la arqueología hidráulica, que él considera el núcleo argumental básico de mi "distorsionada" interpretación. Esta cuestión requiere también algunas matizaciones que de entrada conviene aclarar. Efectivamente tiene razón M. Barceló al afirmar que no he estudiado la huerta de Orihuela, es decir, el agrosistema extenso creado por la canalización de las aguas derivadas de azudes fluviales y situado en el llano aluvial que circunda la ciudad. No era esa mi intención puesto que el "diseño" de este espacio hidráulico (BARCELO, 1989, $X X V)$ no parece ser anterior al siglo $X I$ o, a lo sumo, a mediados del siglo precedente. De lo que se trata en los trabajos recensionados no es de esta huerta urbana, sino de unos agrosistemas anteriores en el tiempo y diferentes en el espacio, que precisamente fueron abandonados -al menos sus áreas de residenciacon la planificación de la huerta de Orihuela. El único lugar donde nos hemos referido -en este caso el plural responde al hecho de ser un trabajo en coautoría con R. Azuar (e. p.)- a la posibilidad de definir el diseño original de la huerta, discriminando sus ampliaciones, fue en el trabajo presentado al Castrum V, que actualmente se halla en curso de publicación.

Para aquellos que estuvieron presentes en dicho coloquio, como es el caso de M. Barceló, debería resultar obvio que su única

\footnotetext{
3 Cfr. el libro La cerámica altomedieval en el sur de al-Andalus, editado por A. MALPICA y publicado en Granada en 1993 , y especialmente para el tema que nos ocupa -el de la identificación de la cerámica emiral correspondiente a sociedades de tradición indígena-, los trabajos de M. Acién, F. Castillo-R. Martínez y el mío propio. Existe además una abundante bibliografía previa: AClÉN ( 1986), ACIÉN y MARTÍNEZ ( 1989), GUTIÉRREZ LLORET (I988 b).
} 
inspiración difícilmente puede constituirla mi propia tesis doctoral, que versa específicamente sobre la problemática histórica de los siglos VII al X en Tudmir. Por contra, en el trabajo del Castrum se distinguieron "dos grandes fases de poblamiento, una anterior al siglo $\mathrm{Xl}$, y otra, establecida a partir de ese momento y fosilizada en el Repartimento de Orihuela en el siglo XIII y caracterizada por la creación de un espacio hidráulico en el territorio dependiente de la ciudad de Orihuela", tal y como indican $\mathrm{H}$. Kirchner y C. Navarro en la nota donde expresan su deseo de que la investigación en esta zona integrara también "la descripción minuciosa de los espacios agrarios cronológicamente discriminados" (KIRCHNER y NAVARRO, 1994, p. 171, not. 27). Precisamente fue esta interesante sugerencia la que motivó mi reflexión acerca de la dificultad de abordar dicha descripción con los métodos de la arqueología extensiva de superficie en la fase de poblamiento - previa a la huerta de Orihuela- que mejor conozco: la emiral. Me he extendido sobre este particular porque no considero correcto ni respetuoso con el trabajo de un colega atribuirme todos los méritos de una reflexión conjunta.

A lo largo de su texto, M. Barceló me atribuye también "dificultades serias" de intelección, desconocimientos o tergiversaciones de ciertos conceptos que utilizo en mis trabajos. No los tengo por tales y creo que todas las referencias conceptuales, propias y ajenas, están convenientemente explicitadas en mis escritos con su pertinente aparato crítico, pese a lo cual volveré nuevamente sobre algunas de ellas. Así, "óptimo agrícola" se aplica en el trabajo de Arbor a "las zonas fértiles de condiciones estables, donde es posible una estructuración más compleja de la comunidad con un máximo de biomasa" (TERRADAS, 1991, p. 91), y donde, en consecuencia, la ocupación agrícola permite "maximizar el rendimiento, o sea la producción" y "regularizar en lo posible el sistema, estabilizándolo" (TERRADAS, 1991, 94); por tanto, "óptimo agrícola" es la relación entre la producción máxima sostenible y los costes de producción, que en el caso del Bajo Segura se sitúa "en las partes más altas de los llanos de inundación, en contacto directo con los piedemontes" (GUTIERREZ LLORET, 1995 a, 79-80) y no en los marjales, también áreas fértiles de alta producción, pero cuyo carácter fluctuante impide la previsión de su mantenimiento. El óptimo agrícola, por tanto, no son las pautas de asentamiento -uillae, fundi, y más tarde qurà-, como M. Barceló parece interpretar, sino las condiciones ambientales que permiten la apropiación de recursos naturales de forma más productiva y estable, mediante las prácticas agrícolas y la tecnología que éstas entrañan.

En esta misma línea de imprecisión conceptual que me atribuye, M. Barceló cuestiona la comprensión y utilización por mi parte del "hermoso y complejo" texto de V. M. Toledo; en primer lugar señala que "una dimensión esencial de la estrategia multiuso del campesino es la utilización de más de una unidad ecogeográfica", que M. Barceló considera completamente ausente de mi "planteamiento general sobre la ocupación de marjales" puesto que únicamente describo "campesinos gestionando un sólo ecosistema, el de los marjales y lagunas". ${ }^{4}$ Pues bien, el medio ambiente transformado por la agricultura (MAT) -conocido a partir de uno de sus elementos tecnológicos: los arcaducesconstituye una unidad geográfica o es "ecogeográficamente" diferente de un ecosistema natural (MAN), aunque éste sea explotado como ocurre en el caso del marjal. ${ }^{5}$ Así, en el trabajo de Arbor se contemplan al menos dos de las unidades geográficas que pudieron gestionar los habitantes de los asentamientos del Bajo Segura. Es lógico suponer que explotaron también otras, como el bosque o la franja litoral, cuyo aprovechamiento no podremos afirmar hasta que sea constatado mediante otras técnicas arqueológicas (palinología, antracología, paleocarpología, arqueozoología, etc.).

La segunda cuestión planteada por $M$. Barceló respecto al texto de $\mathrm{V}$. M. Toledo gira

\footnotetext{
4 M. Barceló, "Acerca de nada...", página 24. Desde ahora, la cursiva en los fragmentos de M. Barceló u otros autores citados corresponde en el texto original a entrecomillados, salvo que se especifique lo contrario.

5 MAT (medio ambiente transformado) y MAN (medio ambiente natural) en terminología de V. M. Toledo, $1993,209$.
} 
en torno a la definición de economía natural, aspecto que de por sí requeriría una extensa reflexión. Efectivamente la economía natural no excluye en principio las prácticas agrícolas, pero como el propio $\mathrm{V}$. M. Toledo señala y $M$. Barceló reproduce "Hay un espectro continuo de combinaciones entre dos tipos extremos de organización social: producción para el uso y producción para el cambio. Cada una de las combinaciones potenciales que pueden hallarse en el modelo corresponde a formas de sociedades específicas históricamente determinadas y aparece a partir de ellas." (TOLEDO, 1993, p. 207). En este espectro de combinaciones y dentro de las formas de producción para el uso, V. M. Toledo distingue entre las "economías naturales" -es decir, "las más simples formas de producción para el uso" (TOLEDO, 1993, p. 207), que pueden o no contemplar la agricultura en el seno de las relaciones ecológicas que establecen con su entorno (por ejemplo, las sociedades cazadoras-recolectoras o las sociedades agrícolas sedentarias respectivamente)- y la "producción campesina" en sentido estricto, en la que intervienen también las relaciones económicas (formas de producción para el cambio), si bien éstas no constituyen la base del mantenimiento y reproducción del productor y su familia (TOLEDO, 1993, p. 207-8).

Desde esta óptica, las formas de producción rural adoptadas por las comunidades campesinas establecidas en el perímetro de los marjales del Bajo Segura entre los siglos VIII y $X$ se parecen más a las definidas por $V$. M. Toledo en el modelo "c" de la figura 3 (Comunidad campesina), que a las del modelo "a" (Caza y recolección en banda), como se inferiría de la única explotación de los marjales. Así, estas comunidades campesinas establecieron flujos con los ecosistemas naturales (los marjales u otros que no conocemos), los ecosistemas transformados por la agricultura de regadío (documentada por los arcaduces) u otros agrosistemas que no podemos discriminar, como podría ser el secano, y, además, desarrollaron formas de intercambio económico, que se constatan, por ejemplo, en la circulación de los primeros productos vidriados procedentes de talleres urbanos como Murcia o Pechina.

Frente a esta forma específica e históricamente determinada de producción rural se constata otro caso social de producción campesina -el de los agricultores del siglo XVIII-, designada por P. Ruiz (1979) como una "peculiar economía campesina", que denota precisamente el cambio hacia formas de agricultura especializada en el marco de la transición al capitalismo. Así, los campesinos de la Edad Moderna explotaban los recursos del baldío sólo en épocas de escasez, subordinando los intercambios ecológicos a la dinámica económica y evidenciando precisamente la simplificación del proceso productivo rural, con la "abolición del flujo de valores de uso" y la integración creciente -y evidentemente impuesta- en el engranaje del mercado (TOLEDO, 1993, p. 207). Quizá la razón por la que M. Barceló no comprende mi texto radica únicamente en que no apostillé la expresión "economía natural" con la de "las de las más simples formas de producción para el uso", al igual que $\mathrm{V}$. M. Toledo, lo que sin embargo no afecta a la intelección de su contenido.

Del mismo modo debo advertir que no hay ninguna equivocación en la cita del artículo de J. Martínez Alier (1993, 22-23), como el lector podrá comprobar; lo que sí está equivocado es el año en el texto de M. Barceló (1989 por 1993 en la versión que manejo). La idea de J. Martínez Alier de que "el medio ambiente es una construcción social" (MARTINEZ ALIER, 1993, p. 23) me pareció sugerente y acertada para subrayar el carácter histórico y cultural de la percepción de un paisaje; de hecho, J. Martínez Alier insiste en ella de forma recurrente en éste y otros trabajos, señalando que "Las relaciones entre la humanidad y la naturaleza son históricas. La percepción y la interpretación de estas relaciones (en lenguajes populares o científicos) también son históricas y, por tanto, la historia ecológica no se puede hacer separadamente de la historia de las ideas de la naturaleza" (MARTINEZ ALIER, 1993, p. 20), o bien "...el medio ambien-

6 BARCELÓ, M.: "Acerca de nada...", p. 23. 
te es una construcción social. Diversas culturas y diversos grupos sociales, en diferentes momentos históricos, se hacen representaciones diferentes de cuáles son y deben ser las relaciones entre los humanos y la naturaleza" (MARTINEZ ALIER, 1994, p. 195).

Tampoco alcanzo a entender la supuesta falta de precisión del concepto "espacio" o "área" perimetral de los marjales. El perímetro de un marjal es, en sentido estricto, el contorno de su superficie, entendida lógicamente como la máxima extensión de la lámina de agua en condiciones normales; por tanto, los espacios perimetrales de los marjales son aquellos que se sitúan fuera o en el límite del área ocupada por el mismo. Tal vez la imprecisión proceda de un uso inapropiado del concepto de perímetro para designar superficies, como se denota en las referencias a "perímetros de irrigación" (BARCELO, 1988, p. 23940), "perímetros irrigados" (KIRCHNER y NAVARRO, 1994, p. 161 y ss., esp. 165) O "diseño del perímetro de riego" (BARCELO, 1989, XVI). Si, en rigor, lo que se me solicita es precisión en la localización topográfica concreta de las cenias volvemos al punto de partida: en los trabajos criticados por M. Barceló proponía una hipótesis sobre el poblamiento y la explotación rural de agrosistemas y ecosistemas naturales, realizada a partir del estudio arqueológico de las áreas de residencia, que son los elementos del asentamiento emiral que hasta el momento he logrado discriminar.

Si se supiera a priori dónde están las norias emirales, seguramente se estarían estudiando los sistemas hidráulicos y no únicamente los arcaduces, pero lo que he tratado de señalar es precisamente los problemas que entraña su localización. Estamos, pues, ante un caso de "asentamiento difuso" inverso al establecido por M. Barceló et alii a partir de la prospección en el término municipal de Felanitx y citado por H. Kirchner y C. Navarro (1993, p. 163): "...se estableció el concepto de los asentamientos difusos cuya realidad más tangible no es el área de residencia sino el espacio irrigado"; es decir, en el caso del asentamiento emiral del Bajo Segura son más tangibles las áreas de residencia que los espacios irrigados, pero esto no impide el reconocimiento de la pauta de asentamiento (BARCELO, 1989, XXXII). Al menos si no lo impide en una dirección, no debería hacerlo en la otra

Me sorpende también que $M$. Barceló no comprenda qué son los "pequeños agrosistemas de regadío de alto rendimiento", puesto que esta apreciación conceptual concuerda con "la opción campesina de una agricultura (...) que da lugar a espacios de cultivo más pequeños, con mayores rendimientos y cultivos mucho más variados" expresada por $\mathrm{H}$. Kirchner y C. Navarro (1993, p. 160). Es evidente que el alto rendimiento se refiere a una alta productividad -definida como la velocidad de producción de biomasa (DUVIGNEAUD, 1978, p. 56)-, obtenida mediante el riego con agua extraída por noria en un espacio que ecológicamente se define como de alta producción. La presunción del tamaño reducido de los sistemas hidráulicos basados en la captación de aguas subterráneas mediante pozos de noria accionados por tracción animal se desprende -aun en el caso de que se asocien formando redes o "campos de norias" y se gestionen de forma comunitaria- de "la estructura espacial de éstos, la fragmentación del aporte de agua, ligado a la fragmentación parcelaria" (CRESSIER, 1995, p. 269), hasta el punto de poder "...funcionar el regadío en cada parcela de forma independiente" como reconoce H. Kirchner (1995, p. 57, not. 13). Así pues, podemos suponer que estos espacios agrarios regados debieron ser pequeños y proporcionar mayores rendimientos que la agricultura no regada, ya que "la producción de los vegetales es proporcional al agua transpirada" (DUVIGNEAUD, 1978, p. 207); precisamente lo que resulta más difícil de suponer es el grado de variedad de los cultivos y esto me obliga a referirme a la noción de agrosistema que tanto consterna a M. Barceló.

"Agrosistema" -expresión menos redundante que la de "agroecosistema" empleada V. M. Toledo y E. Odum- o sistema agrario si se prefiere (CAMPOS y NAREDO, 1980), se refiere al medio ambiente transformado (TOLEDO, 1993, p. 205), es decir, en palabras de E. Odum, "son ecosistemas domesticados que en muchos aspectos se encuentran en una posición inter- 
media entre los ecosistemas naturales, como pastizales y bosques, y los ecosistemas fabricados, como las ciudades" (ODUM, 1995, p. 233) y que se caracterizan, según Odum, además de por tener grandes ambientes de entrada $y$ salida, por ser autótrofos y tener una diversidad reducida en gran medida por el manejo humano, a fin de maximizar el rendimiento (ODUM, 1995, p. 233). Por tanto, la noción de agrosistema tiene por objeto "poner más el énfasis en las relaciones entre elementos que en esos mismos elementos" (PARRA, 1984, p. 260), que, además de la especie o especies cultivadas -como sugiere M. Barceló-, incluyen el suelo, la insolación, las semillas, los subproductos, el trabajo humano y/o animal, los nutrientes y, por supuesto, el agua (la lluvia en el secano y el riego en la huerta), entre otros. Es evidente que no pienso que agrosistema sea lo mismo que "espacio hidráulico", expresión que no dudo en utilizar cuando es pertinente.

En este punto me gustaría retomar lo que M. Barceló considera una "vulneración arbitraria" del sistema conceptual por él elaborado para entender el espacio hidráulico. En primer lugar me veo obligada a rechazar una "invención" que me atribuye; aquella que compete a la "arqueología hidráulica teórica". El calificativo de "teórico" aplicado a la "arqueología hidráulica" no era propio ni gratuito por cuanto considero que el enunciado o establecimiento de unos principios generales -y por tanto fundamentales, ya que constituyen la base sobre la cual se procede y cuyas consecuencias se aplican a toda la disciplina- pertenece al fundamento teórico de la misma, al igual que la definición de un objeto específico y de sus técnicas. El enunciado de principios generales requiere la previa formulación de una hipótesis y su contrastación "utilizando correctamente las prácticas de análisis que se derivan de la comprensión exacta de la noción", como indica el propio M. Barceló a propósito de una aplicación concreta. ${ }^{7} \mathrm{La}$ investigación así producida "puede ser consi- derada como un primer ensayo fundacional de lo que ya puede denominarse arqueología hidráulica" ${ }^{8}$ (KIRCHNER y NAVARRO, 1993, p. 160, citando a M. BARCELÓ, 1992). Al menos así se indica, y de forma contundente, en el trabajo de $\mathrm{H}$. Kirchner y $C$. Navarro, donde además se apostilla: "Pensamos, pues, que las bases teóri$\operatorname{cas}^{9}$ de esta investigación sobre espacios agrarios se encuentran, ahora mismo, sólidamente establecidas" (KIRCHNER y NAVARRO, 1993, p. 160). En consecuencia y conforme a lo expresado por ambas autoras, que forman parte del equipo de M. Barceló, no debería parecerle impropio ni improcedente hablar de una "arqueología hidráulica teórica" o, si así lo prefiere, de una teoría de la arqueología hidráulica, esto es, de sus "bases teóricas".

M. Barceló también señala que mi "supuesta crítica" a los principios generales de la hidráulica andalusí -que yo consideré únicamente matización- se fundamenta en diversos equívocos, consistentes básicamente en la confusión de un problema de método con otro de definición conceptual, patente, siempre según M. Barceló, en mi confusión de los conceptos de "rigidez" o "diseño" con "inmutabilidad" del espacio hidráulico y "estabilidad" con "perdurabilidad" del mismo. ${ }^{10}$ Creo que no hay tal confusión pues la calidad de inmutable, perdurable o durable también se contempla en el sistema conceptual elaborado por $\mathrm{M}$. Barceló y aplicado por el equipo que dirige, siendo precisamente "la rigidesa dels espais hidráulics i la seva durabilidad" la que "els fa especialment aptes per a ser estudiats" (BARCELO, 1992, p. 246). Así, se insiste en la "inmutabilidad de los límites de rigidez que, incluso detrás de una radical transformación de un perímetro irrigado, siempre pueden ser reconocibles" (KIRCHNER y NAVARRO, 1993, p. 160) y, en tanto que mudable también significa que muda, varía o cambia, se entiende que la inmutabilidad de un diseño no es sino un sinónimo de "la invariabilidad del diseño hidráulico inicial" en la que insiste M. Barceló (1989, XXVIII).

\footnotetext{
7 M. Barceló, "Acerca de nada...", página 22 y ss., Vid. supra, not. 4.

8 Vid. supra, not. 4.

9 La cursiva es mía

10 M. Barceló, "Acerca de nada...", página 29 y ss., Vid. supra, not. 4.
} 
Sin duda, en mis trabajos recensionados, especialmente en el relativo a los arcaduces, pretendía tratar un problema de método, que no afectaba a la estabilidad intrínseca de los sistemas hidráulicos, sino a la dificultad -o imposibilidad en ciertos casos- de reconstruir los más antiguos (los de época emiral), situados en los llanos de inundación del Bajo Segura, a partir únicamente de su estudio epidérmico; es decir, mediante las técnicas de campo de la arqueología extensiva, basadas en la prospección superficial y la fotointerpretación. Sin embargo, la interesante y "severa" crítica de M. Barceló me ha sugerido nuevas consideraciones, porque podría decir, parafraseando a J. Arce en una reflexión crítica acerca de las recensiones y a propósito de una aportación de W. Trillmich (ARCE, 1993, p. 24), jcúanto se aprende de M. Barceló -que no es infalible- cuando se tiene que contra-argumentar sus propuestas!.

En efecto, como M. Barceló ha argumentado con claridad "la estructura inicial contiene ya las posibilidades de expansión que pueden preverse"." Ahora bien, como el propio M. Barceló señala más adelante en su artículo de réplica, recogiendo reflexiones anteriores (BARCELÓ, 1989, XXV y XVIIII), "La rigidez de los espacios hidráulicos viene determinada por la exigencia de la gravedad para hacer circular el agua, lo cual impone límites rígidos al espacio hidráulico y una capacidad de crecimiento o ampliación limitada. La localización de la captación, su caudal y las pendientes del terreno determinan la extensión y morfología del sistema"; '12 por tanto, si se mantienen estas condiciones iniciales -captación, caudal y pendientes- el "diseño" del sistema será "durable", como dice M. Barceló (1992, p. 246), y se podrá "discernir lo nuevo de lo viejo".'33 Lo que se está planteando, por tanto, es la posibilidad de distinguir las etapas de crecimiento, las sucesivas ampliaciones de un espacio regado por su estratigrafía horizontal, ${ }^{14}$ mediante la metodología que ha desarrollado el equipo de investigación dirigido por M. Barceló con positivos resultados.

Sin embargo, la sedimentación puede modificar de forma difícilmente previsible la topografía y, por tanto, alterar las pendientes y distorsionar la exigencia de gravedad inicialmente prevista (este es el sentido de la observación de Mossen Bellot citada en mi trabajo ${ }^{15}$ ). Desde este punto de vista, los espacios hidráulicos no sólo admiten su destrucción, como indica M. Barceló (1989, XVIII), sino también la estratificación -en este caso vertical- al menos en las "superfícies d'activa acumulació al.luvial durant les revingudes periòdiques i recurrents", principal mecanismo morfogénico de los llanos de inundación (MATEU, 1989, p. 170); esta posibilidad de crecimiento vertical -que no debe confundirse con una "catástrofe natural irreversible", puesto que es recurrente, periódica y a menudo aprovechada con finalidad agrícola- ${ }^{16}$ puede

I M. Barceló, "Acerca de nada...", página 22

12 M. Barceló, "Acerca de nada...", página 30

13 M. Barceló, "Acerca de nada...", página 22

14 Y entiendo que M. Barceló se refiere a ésta -la estratigrafía horizontal- cuando afirma: "Però cal dir també que cap horta de l'est peninsular ha estat objecte d'estudi arqueològic. Els estudis geogràfics, tal com s'han fet fins ara, no condueixen a entendre l'estratigrafia -si és que n'hi ha- de l'horta. El que, doncs, fa molt difícil integrar-les en l'actual discussió sobre l'hidraulisme andalusí excepte com a soroll especulatiu" (BARCELO, 1993, 50).

15 "Redován o Aben Reduán (como dice el libro de los Repartimientos) fue siempre poseído por los Mirón (...) hasta el año 1490 , que lo compró don Jaime Santángel. No era más de una grande alquería, y debía ser harta mejor tierra de lo que es hoy, porque la cequia de Escorratel iba por mucho más arriba; pero con las avenidas de la rambla crece y se alza la tierra y decrece la huerta faltándole la agua" (BELLOT, 1956, II, I8I). Mosén Bellot recopila y transcribe a principios del siglo XVII mucha de la documentación bajomedieval del archivo de Orihuela

16 Las aguas de avenida, de alfayt en el repartimiento murciano, han sido aprovechadas tradicionalmente para el riego en las regiones subáridas (MORALES, 1969-69). Su interés radica no sólo en el aporte hídrico sino también en el enorme poder fertilizante de las "aguas turbias", cuyos légamos mejoran los suelos sobre los que se depositan, como señaló Cavanilles (I797, II, 255) a propósito de Agost, hasta el punto de ser "increible lo que vale semejante abono, preferible por muchos aspectos al de los estiércoles" (MUSSO y FONTES, 1847, I56, cit. por R. Pocklington $(1989,380)$. El interés por el "riego de turbias" originó en las huertas de Lorca, Murcia y otras localidades del sureste de la Península lbérica, un sistema de aprovechamiento muy complejo del riego eventual, en el que "las aguas 'turbias', tan beneficiosas para las tierras, correrían libremente por las boqueras y regajos permitiendo la extensión de los cultivos..." (CALVO GARCIA-TORNEL, 1968-69, 124). Así, las avenidas se integraban en el sistema agrícola, percibiéndose únicamente como catastróficas cuando "...eran excesivas para la capacidad de absorción del sistema, era éste pronto destruido y el agua volvía a buscar su antiguo curso", inundando a su paso poblaciones y terrenos (CALVO, 1968-69, I24). 
afectar a todo el sistema hidráulico o parte del mismo que, llegado el caso, deberá ser replanteado, sin que esto implique necesariamente una ampliación o afecte con exclusividad "a los aterrazamientos o a la forma de algunas parcelas". ${ }^{17}$ Por tanto, aunque se mantenga el uso continuado de un sistema hidráulico y sea estable su gestión social, si cambia "la exigencia de la gravedad para hacer circular el agua" y ésta determina la rigidez del sistema ¿cambia el sistema?. Creo que esta cuestión sí puede afectar al enunciado de principios generales y consecuentemente también a los métodos y técnicas de la arqueología hidráulica, que, al menos hasta ahora, no ha contemplado en la práctica la posibilidad de la estratificación vertical y sus eventuales consecuencias en los sistemas hidráulicos.

No obstante, como señalé con anterioridad, esta apreciación personal no pretendía vulnerar un sistema conceptual que ha producido resultados positivos, ni negar las posibilidades de la arqueología en el análisis de los sistemas hidráulicos, suficientemente probada por los trabajos de M. Barceló, su equipo, y otros investigadores como $P$. Cressier o A. Malpica, sino todo lo más enriquecerlo si resultaba ser acertada. Por esta razón, creo que no tiene sentido volver ad infinitum -y yo no pienso hacerlo- sobre una cuestión cuya resolución radica en última instancia en el análisis práctico, esto es, en "pasar por la gran prueba de analizar un perímetro de los tradicionalmente conocidos como huertas, ${ }^{18}$ mucho más extensos y con caudales de agua en circulación muy importantes", que reclamaba -y aún falta- $M$. Barceló en 1988 (BARCELÓ, 1988, p. 255), y, añadiría yo, situado en un llano de inundación, como es el caso de la huerta de Orihuela a más de las de Murcia y Valencia.

Para concluir esta contrarréplica, cuya extensión el lector disculpará por la necesidad de dar cumplida respuesta a las densas reflexiones de M. Barceló, quisiera referirme a la que considero, con mucho, la observación más pertinente de su artículo "Acerca de nada...". Ciertamente no otorgo ninguna categoría científica a un fetiche y no considero los arcaduces tales; precisamente lo que pretendía con ese "arrebatado colofón" era puntualizar la consideración de fetiche que se desprendía de una intervención oral del propio M. Barceló en el coloquio sobre Acculturazione e Mutamenti, celebrado en Siena en 1993, donde ante estos arcaduces mostrados por mí planteó la necesidad imperiosa de que los arqueólogos dejásemos de estudiar fetiches. Para evitar la confusión debería haber escrito "concedamos a los conocimientos históricos que se establecen a partir del estudio de estos arcaduces cerámicos una cualidad científica que no tienen los fetiches y que no les otorgan aque"los que por tales los toman". No obstante, no lo escribí y como lo que "escrito está, escrito queda" reconozco y agradezco desde aquí, esta importante matización de $M$. Barceló, que contribuye a aquilatar enormemente mi reflexión.

En último lugar quisiera señalar, para tranquilidad de M. Barceló, que en mis textos, con toda su humildad, hay muchos autores innominados, algunos que todos pueden identificar puesto que son mis maestros y por tales los tengo, y otros muchos, los más, que ni siquiera yo identifico, porque forman parte de lecturas, conversaciones y enseñanzas entretejidas en numerosos discursos, que pasan de mano en mano y enriquecen "ese colectivo que es la ciencia", como bellamente expuso Ricardo Olmos, recreando quizá o reconstruyendo, a José Luis López Aranguren (OLMOS, 1993, p. 23).

\footnotetext{
17 M. Barceló, "Acerca de nada...", página 31

18 Vid. supra, not. 4: la cursiva equivale a un entrecomillado en el original.
} 


\section{BIBLIOGRAFÍA}

ACIÉN ALMANSA, M. (1984): "La formación y destrucción de al-Andalus". $H^{a}$ de los Pueblos de España. Tierras fronterizas (I), Andalucía, Canarias. Barcelona.

ACIÉN ALMANSA, M., (1986): "Cerámica a torno lento en Bezmiliana. Cronología, tipos y difusión", I Congreso de Arqueología Medieval Española (Huesca, 1985), IV, pp. 243-267, Zaragoza.

ACIÉN ALMANSA, M. (1989): "Poblamiento y fortificación en el sur de al-Andalus. La formación de un país de Hușūn", III Congreso de Arqueología Medieval Española, (Oviedo, 1989), I, pp. I35- I50, Oviedo.

ACIÉN ALMANSA, M. (1994): Entre el Feudalismo y el Islam. CUmar Ibn Hafșūn en los historiadores, en las fuentes y en la historia, Universidad de Jaén.

ACIÉN ALMANSA, M. (e. p.): "Poblamiento indígena en al-Andalus e inicios del primer poblamiento andalusi", El siglo VIII. Islam y Occidente, un primer encuentro (Alcalá de Henares-Madrid, 1993).

ACIÉN ALMANSA, M. y MARTÍNEZ MADRID, R. (1989): "Cerámica islámica arcaica del sureste de alAndalus", Boletín de Arqueología Medieval, 3, pp. 123-135.

ARCE, J., (1993): "Arqrítica, autocrítica y los enfrentamientos innecesarios", Arqrítica, 5, pp. 23-4.

AZUAR, R. y GUTIÉRREZ, S., (e. p.): "Formación y transformación de un espacio agrícola islámico en el sur del País Valenciano: el Bajo Segura (siglos IX-XIII)", Castrum V (Murcia, 1992)

BARCELÓ PERELLÓ, M., (1988): "La arqueología extensiva y el estudio de la creación del espacio rural", Arqueología medieval en las afueras del medievalismo, M. BARCELÓ et alii, Barcelona, pp. 195-274.

BARCELÓ PERELLÓ, M., (1989): "El diseño de espacios irrigados en al-Andalus: un enunciado de principios generales", I Coloquio de Historia y Medio Físico; el agua en zonas áridas: arqueología e historia (Almería, 1989), XIII-L, Almería.

BARCELÓ PERELLÓ, M., (1992): Quina arqueologia per al-Andalus?. Coloquio Hispano-Italiano de Arqueología Medieval (Granada, 1990), pp. 243-252, Granada.

BARCELÓ PERELLÓ, M., (1993): "Arqueologia hidràulica i arqueologia medieval: encara mes consideracions des de les afores del medievalisme", IV Congreso de Arqueología Medieval Española (Alicante, 1993), I, pp. 49-55.

CALVO GARCÍA-TORNEL, F., (1968-69): "La huerta de Murcia y las avenidas del Guadalentín", Papeles del Departamento de Geografía, I, Universidad de Murcia, pp. III-137.

CAMPOS, P. y NAREDO, J. M., (1980): "La energía en los sistemas agrarios", Agricultura y Sociedad, 15, pp. 17-I I3.

CAVANILLES, J. A., ( 1797): Observaciones sobre la historia natural, geografia, agricultura, población y frutos del Reyno de Valencia, Madrid (reimp. facs. Valencia, 1989).
CHALMETA, P., (1988): "La sociedad andalusí, la economía y las instituciones", Historia General de España y América, III, pp. 459-544, Rialp, Madrid.

CRESSIER, P., (1995): "Hidráulica rural tradicional de origen medieval en Andalucía y Marruecos. Elementos de análisis práctico", El agua. Mitos, ritos y realidades, J. A. GONZÁLEZ y A. MALPICA (coords.), Granada, pp. 255-286.

\section{DUVIGNEAUD, P., (1978): La síntesis ecológica, Madrid.}

GUICHARD, P., (1976): Al-Andalus. Estructura antropológjca de una sociedad islámica en Occidente, Barcelona.

GUICHARD, P., (1980): "La Valencia musulmana", Nuestra Historia, 2, pp. 202-28I.

GUICHARD, P., (1985): "El Islam alicantino", Historia de la Provincia de Alicante, III, pp. 57- I70, Alicante.

GUTIÉRREZ LLORET, S. (1988 a): Cerámica común paleoandalusí del sur de Alicante (siglos VII-X). Alicante.

GUTIÉRREZ LLORET, S. (1988 b): "El poblamiento tardorromano en Alicante a través de los testimonios materiales: estado de la cuestión y perspectivas", Antigüedad y Cristianismo (Murcia), V, pp. 323-373.

GUTIÉRREZ LLORET, S. (1992): "Espacio y poblamiento paleoandalusí en el sur de Alicante: origen y distribución". III Congreso de Arqueología Medieval Española (Oviedo, 1989), pp. 34I-348, Oviedo.

GUTIÉRREZ LLORET, S. (1993 a): "De la ciuitas a la madina: destrucción y formación de la ciudad en el sureste de al-Andalus. El debate arqueológico", IV Congreso de Arqueología Medieval Española (Alicante, 1993), I, pp. I3-36.

GUTIÉRREZ LLORET, S. (1993 b): "La cerámica paleoandalusí del sureste peninsular (Tudmīr): producción y distribución (siglosVII al X)", La cerámica altomedieval en el sur de al-Andalus (Salobreña, 1990), pp. 37-66, Granada.

GUTIÉRREZ LLORET, S. (1994): "La formación de Tudmir desde la periferia del Estado islámico", II jornadas sobre Madinat al-Zahrā': al-Andalus antes de Madinat alZahrā' (Cordoba, 1991), Cuademos de Madīnat al-Zahrä', 3 (|99|), pp. 9-21.

GUTIÉRREZ LLORET, S., (1995 a): "El origen de la huerta de Orihuela entre los siglos VII y Xl: una propuesta arqueológica sobre la explotación de las zonas húmedas del Bajo Segura", Arbor (Madrid), mayo, № 593, pp. 65-94.

GUTIÉRREZ LLORET, S. (1995 b): "La experiencia arqueológica en el debate sobre las transformaciones del poblamiento altomedieval en el SE. de al-Andalus: el caso de Alicante, Murcia y Albacete", Aculturazione e mutamenti. Prospettive nell'archeologia medievale del Mediterraneo (Siena, 1993), Firenze, pp. 165-189.

GUTIÉRREZ LLORET, S. (e. p.): "Ciudades y conquista: el fin de las ciuitates visigodas y la génesis de las mudun islámicas en el sureste de al-Andalus", La ciudad islámica en la Alta Edad Media (Granada, 1995) 
KIRCHNER, H. y NAVARRO, C., (1994): "Objetivos, métodos y práctica de la arqueología hidráulica", Arqueología y territorio medieval, I, Jaén, pp. I59-182.

KIRCHNER, H., (1995): "Construir el agua. Irrigación y trabajo campesino en la Edad Media", Arbor (Madrid), mayo, No 593, pp. 35-64.

MANZANO MORENO, E., (1993): "El asentamiento y la organización de los ŷund-s sirios en al-Andalus", AlQanțara, XIV, fasc. 2, pp. 327-359.

MANZANO MORENO, E., (e. p.): "Arabes, bereberes e indígenas: al-Andalus en su primer periodo de formación", L'incastellamento: confronto fra società feudale e non feudale nel Mediterraneo occidentale (Roma, 1994).

MARTÍNEZ ALIER, J., (1993): "Temas de historia económico-ecológica", Ayer, II, pp. 19-48.

MARTÍNEZ ALIER, J., (1994): De la economía ecológica al ecologismo popular, $2^{\mathrm{a}}$ ed., Barcelona.

MATEU BELLES, J. F., ( 1989): "Assuts i vores fluvials regades al País Valencià Medieval", Los Paisajes del agua. Lobro jubilar dedicado al profesor A. López Gómez, Universitat de València-Universitat d'Alacant.

MORALES GIL, A., (1968-69): "El riego con aguas de avenida en las laderas subáridas", Papeles del Departamento de Geografia, I, Universidad de Murcia, pp. I67-183.
MUSSO y FONTES, J., ( 1847$)$ : Historia de los riegos de Lorca, Murcia (reimp. Lorca, 1982).

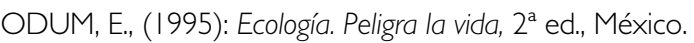

OLMOS, R., (1993): "La ilusión de investigar", Arqrítica, 5, 22-23.

PARRA, F., (1984): Diccionario de ecología, ecologismo y medio ambiente, Madrid.

POCKLINGTON, R., ( 1989 ): "observaciones sobre el aprovechamiento del agua torrencial para la agricultura en Murcia y Lorca durante la época árabe", Murcia Musulmana, pp. 375-85, Murcia.

RUIZ TORRES, P. M., (1979): "Propiedad de la tierra y estructura de clases en el campo valenciano durante los siglos XVIII y XIX: los carrizales de Elx", Estudis d'Història Comtemporània del País Valencià, I, pp. 75-I34.

SCHIØLER, T., ( 1973): Roman and Islamic water-lifting Wheels, Lund.

TERRADAS, J., (|99|): Ecología, hoy, 8ª ed., Barcelona.

TOLEDO, V. M., (1993): "La racionalidad ecológica de la producción campesina", Ecología, campesinado e Historia, E. SEVILLA y M., GONZÁLEZ (eds.), pp. 197-218, Madrid. 